\title{
A flip-chip LIGA assembly technique via electroplating
}

\author{
L.-W. Pan, L. Lin, J. Ni
}

\begin{abstract}
A novel flip-chip assembly technique by means of electroplating at a low processing temperature has been developed. Mesoscopic gears, made of nickel with $1500 \mu \mathrm{m}$ in diameter and $250 \mu \mathrm{m}$ in height, have been bonded to glass substrate. The bonding process is conducted by nickel electroplating at $50{ }^{\circ} \mathrm{C}$ with a current density at $53.3 \mathrm{~A} / \mathrm{m}^{2}$. The nickel electrolyte is found to penetrate about $100 \mu \mathrm{m}$ from the perimeter to the center of the gear and form a good bond. This process can be applied to the integration of high-aspect-ratio microstructures with substrates that have pre-fabricated microelectronics massively and in parallel. As such, it provides a powerful way to achieve the integration of meso- and microscopic electromechanical systems.
\end{abstract}

\section{Introduction}

High-aspect-ratio structures have been fabricated by many methods such as LIGA, laser or micro-EDM [1-3]. Their manufacturing processes are not compatible with IC (Integrated Circuit) fabrication such that the integration of these microstructures with microelectronics has been a challenge that has hindered the advance of the field. Several methods have been proposed for the device integration. For example, cold welding by using indium has been successfully demonstrated to bond HexSil [4] polysilicon structures with microelectronics but the bonding strength is weak [5]. Flip-chip bonding has been used to bond MEMS chiplets to electronics [6] on a chip to chip level but not on the device level. Polymer bump has been used as

Received: 7 July 1999/Accepted: 30 June 2000

L.-W. Pan ( $₫)$, J. Ni

Mechanical Engineering, University of Michigan, CoE/2250 66 Brown Lab., 2350 Harward St, Ann Arbor, MI 48109-2125, USA

L. Lin

Mechanical Engineering, University of California,

5126 Etcheverry Hall, Berkeley, CA 94720-1740, USA

The authors wish to thank Dr. G. He for his assistance in taking SEM microphotos and Drs. D. Plummer, E. Garcia and A. Oliver from Sandia National Laboratory for providing LIGA

microstructures. This project is supported in part by a NSF award (DMI-0096220) and a DARPA award under the MTO/MEMS program.

This paper was presented at the Third International Workshop on High Aspect Ratio Microstructure Technology HARMST'99 in June 1999. the bridge for integration [7]. However, the long term stability of polymer material is generally poor. Recently, diffusion bonding has been applied in a LIGA integration process [8]. The high temperature requirement for diffusion bonding limits potential applications.

As the manufacturing technologies of high-aspect-ratio microstructures become mature and diversified, electromechanical integration will be complicated but inevitable. Therefore, a versatile integration process is attractive. This paper presents a flip-chip assembly technique by electroplating at a low processing temperature with good bonding strength. We believe this technology can be applied to a broad range of integration applications massively and in parallel.

\section{Bonding process}

In order to demonstrate the concept of bonding by electroplating, a simple experimental setup is designed as shown in Fig. 1. The glass substrate at the bottom is used as the "microelectronic substrate" and the second glass substrate on the top acts as the finished "LIGA substrate" that has microstructures attached to it. A $\mathrm{Cr} / \mathrm{Au}$ layer of $500 / 2000 \AA$ in thickness is deposited as the seed layer on the microelectronics substrate. Mesoscopic gears fabricated by the LIGA process [1] are then placed on top of the microelectronics substrate and the LIGA substrate is put on top of the gears as shown in Fig. 1a. The whole assembly is then put into nickel electrolyte that has been added with a wetting agent to reduce the surface tension of the liquid [9]. Electroplating process is conducted at $50{ }^{\circ} \mathrm{C}$ with current density of $53.3 \mathrm{~A} / \mathrm{m}^{2}$. Figure $1 \mathrm{~b}$ applies after $80 \mathrm{~min}$ of the electroplating process. A layer of nickel is deposited on the microelectronics substrate with a thickness of about $2 \mu \mathrm{m}$.

Excessive deposition is found on the side-walls of the gear as well as on the perimeter of both top and bottom interfaces of the gear and glass substrates. The bonding strip, due to excessive nickel deposition, is formed between the bottom surface of the gear and microelectronics substrate. The bonding strip on the top surface of the gear forms a weak bond to the LIGA substrate such that the glass can be easily removed as shown in Fig. 1c. If a real LIGA substrate is used, the seed layer of copper used to connect the microstructure and LIGA substrate can be selectively etched away to complete the process [10].

\section{Experimental results}

Two gears, $1500 \mu \mathrm{m}$ in diameter, are separated $10 \mathrm{~mm}$ apart during the parallel bonding process via electroplat- 

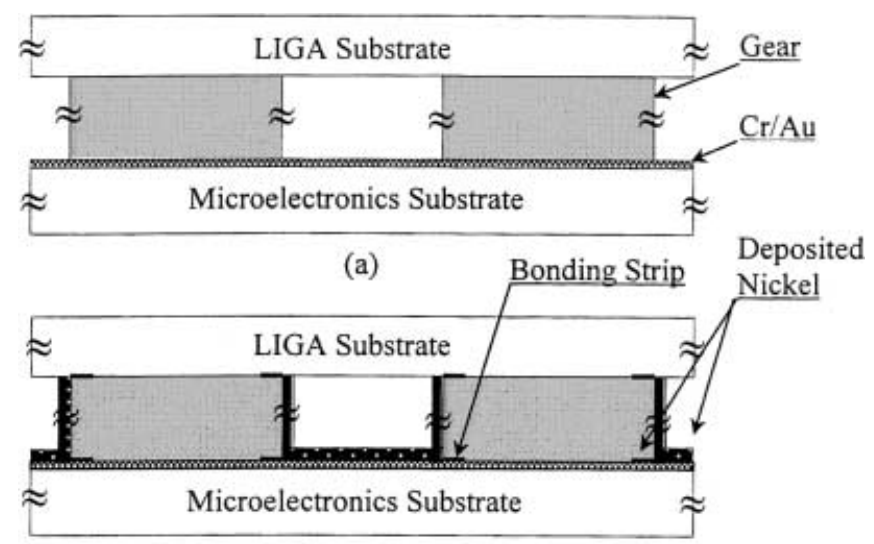

(b)

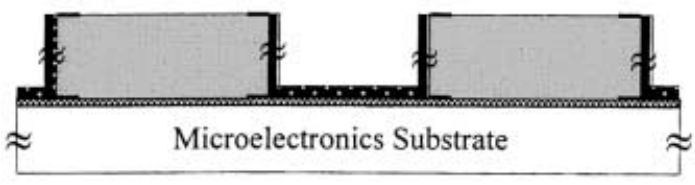

(c)

Fig. 1. Bonding Process

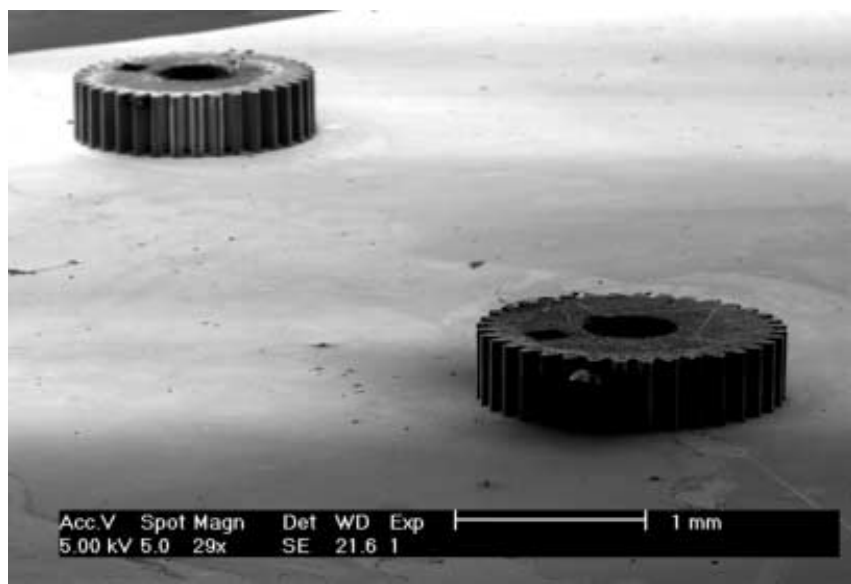

Fig. 2. The SEM microphoto of two bonded gears

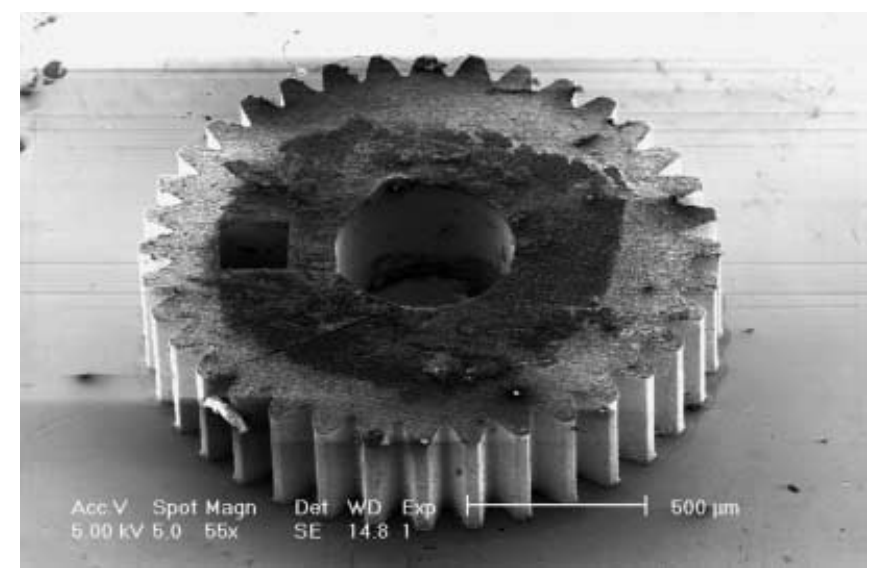

Fig. 3. A single gear with $1.5 \mathrm{~mm}$ in diameter. The gear contour does not change during the process ing. After $80 \mathrm{~min}$, both of them are bonded to a glass substrate as shown in Fig. 2. The bonding strength is strong and both gears remain intact with the substrate after repetitively poking them by using probes on a probe-station. Figure 3 shows the close view SEM micrograph of one gear. The newly-deposited nickel can be barely seen at the top of the gear as well as the interface between the gear and the

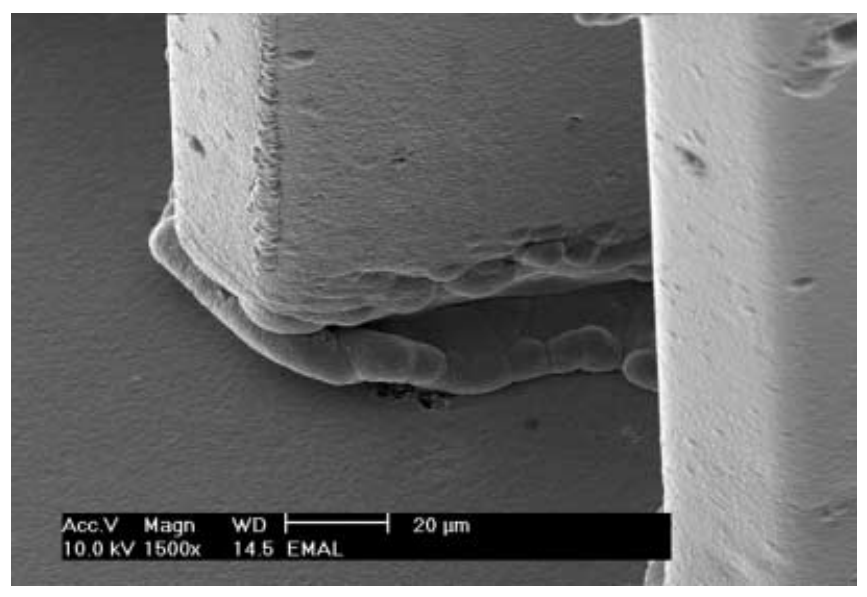

Fig. 4. The bonding interface after $20 \mathrm{~min}$

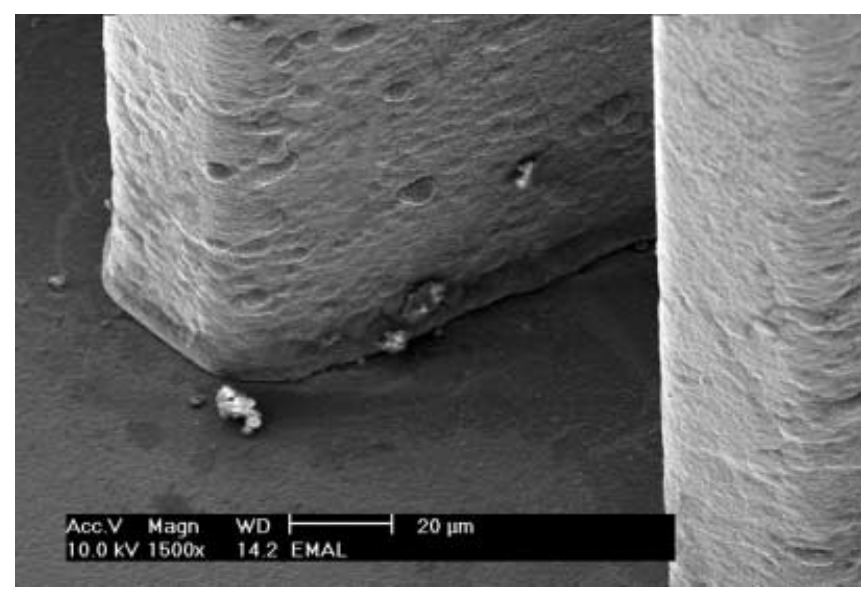

Fig. 5. The bonding interface after $40 \mathrm{~min}$

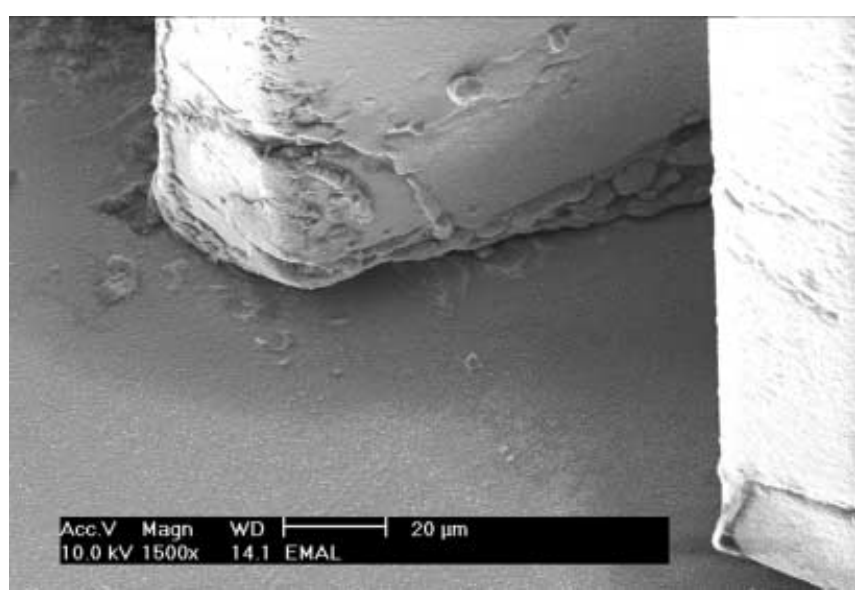

Fig. 6. The bonding interface after $60 \mathrm{~min}$ 
microelectronics substrate. The original contour of the microgear does not change during the bonding process.

In an effort to optimize the bonding process, four gears are bonded to substrates with processing time of 20, 40, 60 and $80 \mathrm{~min}$. Figures $4-7$ show bonding interfaces at the

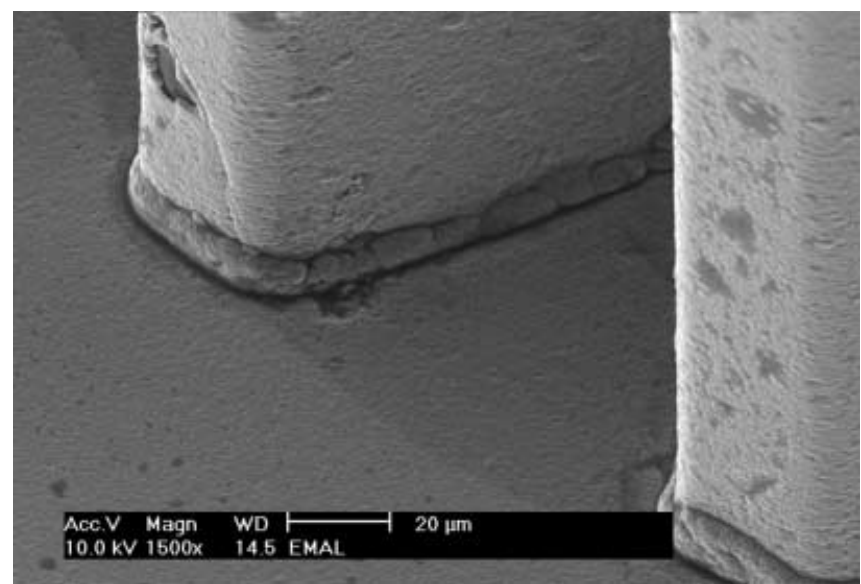

Fig. 7. The bonding interface after $80 \mathrm{~min}$

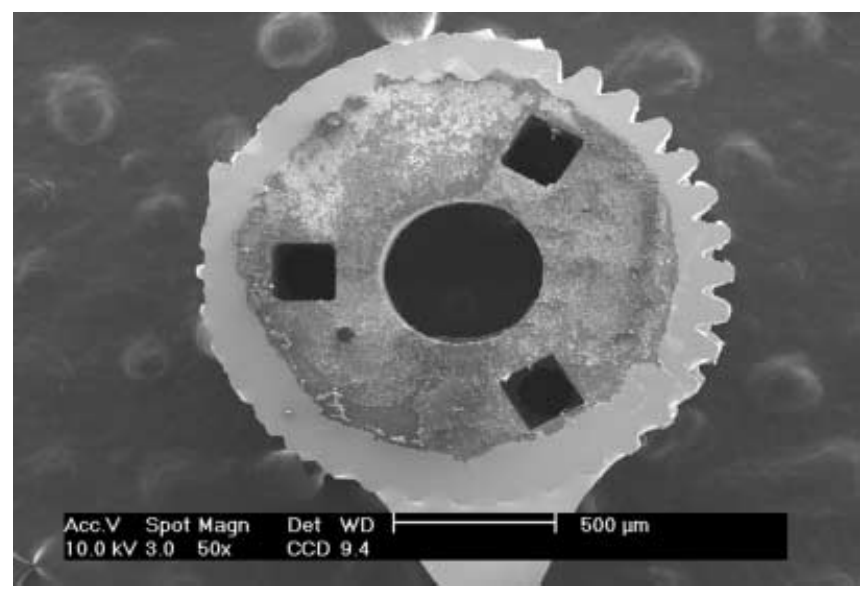

Fig. 8. The SEM microphoto showing the bonding interface on the gear after the separation

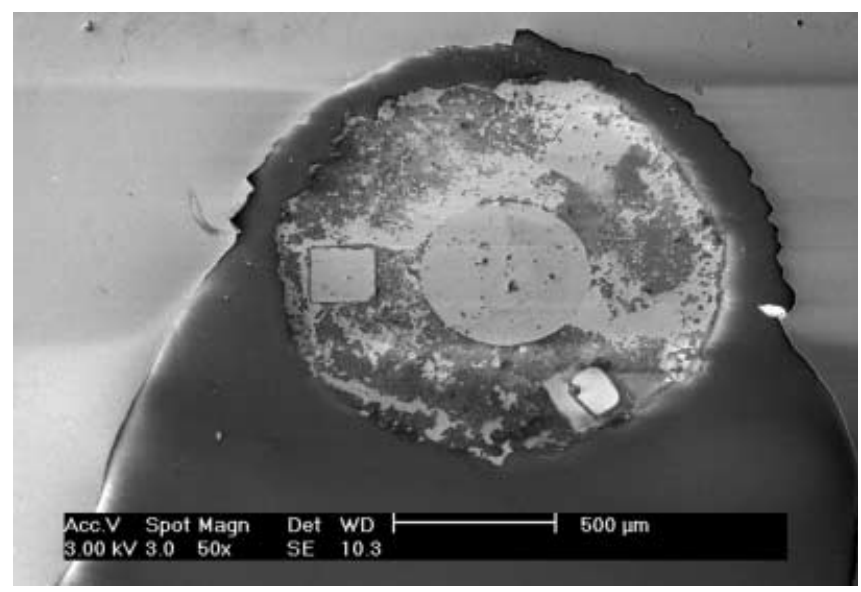

Fig. 9. The SEM microphoto showing the peeled-off region on the glass substrate end of these experiments, respectively. After the first $20 \mathrm{~min}$, small nickel grains are seen on the side-wall as shown in Fig. 4. The surface becomes rougher than those newly-fabricated LIGA microstructures. Furthermore, nickel is observed to grow faster at the sharp edges of gears due to stronger electrical field. After $40 \mathrm{~min}$, the surface becomes rougher as shown in Fig. 5. Nickel deposition on the bottom of gear begins to merge with nickel deposition from the substrate. At the end of $60 \mathrm{~min}$, the merge of nickel deposition is stronger and the side wall seems to regain the smoothness as seen in Fig. 6. At the completion of the bonding process after $80 \mathrm{~min}$, Fig. 7 applies. The bottom of the gear teeth has a ring-shape structure with diameter of about $5 \mu \mathrm{m}$ and the side-wall surface is relatively flat. The newly-deposited nickel is estimated to have a thickness of about $2 \mu \mathrm{m}$.

\section{Discussions}

In order to examine the bonding strength, the bonded structures were pulled off the substrate by tweezers. Figures 8 and 9 are the gear and the peeled-off glass substrate under SEM, respectively. The electroplating technique appears to provide a stronger bond than the

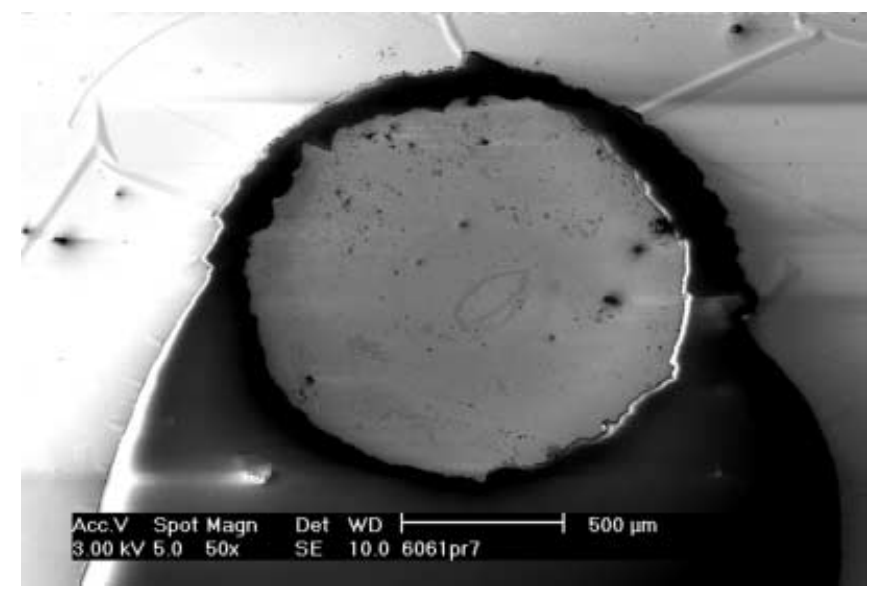

Fig. 10. After $30 \mathrm{~min}$ of nickel etching, gold layer shows up from the substrate in Fig. 9

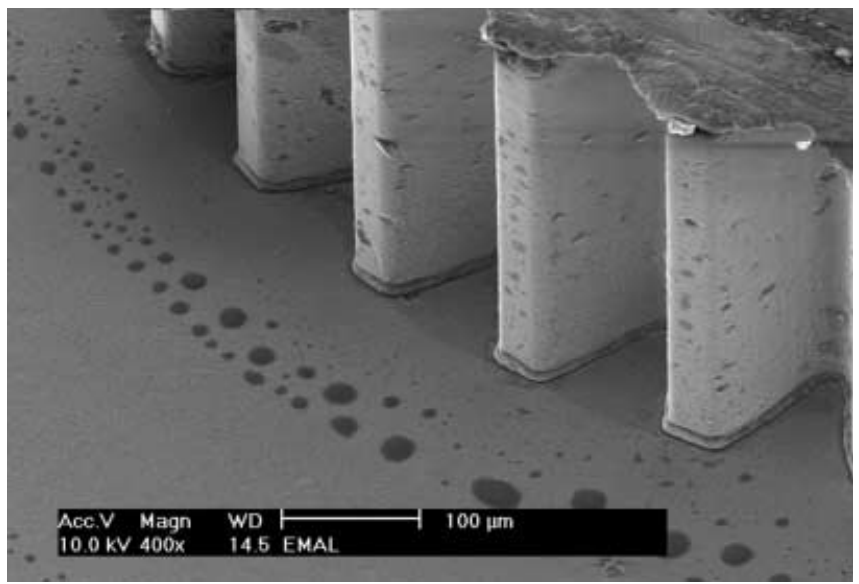

Fig. 11. The SEM microphoto showing the excessive deposition on top of the gear 
adhesion force between the seed layer of chromium to the glass wafer such that some of the seed layer were peeled off. Figure 8 also reveals that the good bonding area is limited to about a $100 \mu \mathrm{m}$-wide strip along the perimeter of the gear and the rest of the surface did not have a complete bond. By conducting a profile scan on the surface of Fig. 9, it is found that the thickness of newly-deposited nickel is about $2 \mu \mathrm{m}$ on top of the gold layer. After removing all the nickel layer by immersing the glass substrate in nickel etchant for $30 \mathrm{~min}$, the shining color of gold shows up again in Fig. 10. Therefore, it can be concluded that the messy surface in Fig. 9 is caused by residual nickel.

Many factors will affect the size of the bonding strip in the bonding process, including the surface roughness at the bottom of the gear, diffusivity and concentration of nickel electrolyte, and current density in the electroplating process. Rough surface, high diffusivity and concentration of nickel electrolyte, and low current density will assist the diffusion of ion and help increasing the width of the bonding strip. It is believed that these steps will increase the bonding strength. The pulse plating technique [11] may be used to help the ion diffusion process for better penetration and to improve the bonding strength.

It appears that nickel has difficulty to bond with glass such that no bonding can be identified on the top surface. However, electroplating does occur at the top surface, especially for places that have sharp edges. Shown in Fig. 11 is the excessive deposition at the top surface of the gear. This unwanted deposition sometimes covers a great portion of the space between two gear teeth. This problem may be solved if the ion diffusion process into the gaps between the LIGA gear and the top glass substrate is limited or a true LIGA substrate is used [10].

\section{Conclusions}

The concept of flip-chip LIGA assembly via electroplating is introduced and demonstrated. Experimental results show that strong bonding is achieved by pressing two conductive surfaces together while the electroplating process is conducted. The optimal processing time is found at about $80 \mathrm{~min}$ for nickel electrolyte under a current density at $53.3 \mathrm{~A} / \mathrm{m}^{2}$ to bond LIGA gears with $\mathrm{Cr} / \mathrm{Au}$ seed layer on a flat glass substrate. During the bonding process, a $2 \mu \mathrm{m}$ thick, new nickel layer is deposited. The bonding is formed on a $100 \mu \mathrm{m}$-wide doughnut-shape strip at the interface of the gear and microelectronics substrate from the outside edge of the gear toward the center. It is concluded that rough surface, high diffusivity of nickel electrolyte and low current density will help the bonding process. This new technique is expected to provide an alternative way in the massively parallel integration of LIGA structures and microelectronics.

\section{References}

1. Bacher W; et al (1995) The LIGA technique and its potential for microsystems - A survey. IEEE Trans. on Industrial Electronics 42: 431-441

2. Bloomstein TM; Ehrlich DJ (1991) Laser-chemical Threedimensional Writing of Multimaterial Structures for Microelectromechanics. Proceedings of the 1991 IEEE Micro Mechanical Systems - MEMS '91, pp. 202-203

3. Langen HH; et al (1995) Self-aligned machining and assembly of high aspect ratio microparts into silicon. Proceedings of the IEEE Micro Electro Mechanical Systems 1995, pp. 250-255

4. Keller C; Ferrari M (1994) Milli-scale polysilicon structures. Proceedings of Solid-State Sensor and Actuator, Hilton Head Island, pp. 132-137

5. Singh A; et al (1997) Batch transfer of microstructures using flip-chip solder bump bonding. Proceedings of the 1997 International Conference on Solid-State Sensors and Actuators. Part 1 (of 2): 265-268

6. Koester D; et al (1994) Multi-User MEMS Processes (MUMPS) Introduction and Design Rules, rev. 3, MCNC MEMS Technology Applications Center, 3021 Cornwallis Road, Research Triangle Park, NC, 27709

7. Oh KW; Ahn CH (1998) Development of an innovative flipchip bonding technique using micromachined conductive polymer bumps. Proceedings of Solid-State Sensor and Actuator Workshop Hilton Head Island, pp. 170-173

8. Christenson TR; Schmale DT (1998) A batch wafer scale LIGA assembly and packaging technique via diffusion bonding. Proceedings of IEEE International Conference on MEMS, pp. 476-481

9. MacDermid, Inc., 245 Freight Street, Waterbury, CT 06702

10. Pan L; Lin L (2000) Batch transfer of LIGA microstructures by selective electroplating and bonding. Proceedings of IEEE Micro Electro Mechanical Systems Workshop, pp. 259-264

11. Romankiw LT; Palumbo TA (1987) Electrodeposition in the Electronic Industry. Proceedings of the Symposium on Electrodeposition Technology, Theory and Practice, pp. 13-41 